\title{
Spatiotemporal variations of cultivated land use efficiency in the Yangtze River Economic Belt based on carbon emission constraints
}

\author{
LUO Xiang ${ }^{1}$, AO Xinhe ${ }^{1}$, ZHANG Zuo ${ }^{1}$, "WAN Qing ${ }^{2}$, LIU Xingjian ${ }^{3}$ \\ 1. College of Public Administration, Central China Normal University, Wuhan 430079, China; \\ 2. School of Management, Wuhan Institute of Technology, Wuhan 430205, China; \\ 3. Department of Urban Planning and Design, University of Hong Kong, Hong Kong 999077, China
}

\begin{abstract}
In this study, the carbon emissions (CEs) from cultivated land (CL) were included as an undesirable output in the utilization efficiency of such land. A slack-based model was used to calculate the CL use efficiency (CLUE) for 11 provinces and cities in the Yangtze River Economic Belt (YREB) from 2007 to 2016, and then a kernel density estimation map was drawn to analyze the spatiotemporal variations of CLUE. The Tobit model was also employed to analyze the factors affecting the CLUE. The results show the following. 1) In the YREB, the CEs from $C L$ showed a rising and then a slowly decreasing trend. In this paper, we calculate CEs by carbon emission factors and major carbon sources, and the CEs from CL in the YREB totaled 25.2354 million tons in 2007. By 2014, the value had increased gradually to 28.4400 million tons, and by 2016 it had declined to 27.8922 million tons, suggesting that the carbon-emission reduction measures of the government had an impact. 2) The CLUE of various provinces and cities in the YREB showed an upward trend in the time dimension, while for the spatial dimension, the kernel density was high in the east and low in the west, and the areas with high kernel density were mainly located in the Yangtze River Delta. 3) The per capita gross domestic product, the primary industrial output, and the number of agricultural technicians per 10,000 people had positive effects on the CLUE. The CL area per capita and the electrical power per hectare for agricultural machinery had significant negative impacts on CLUE. In addition, every $1 \%$ increase in the number of agricultural technicians increased the CLUE by $0.057 \%$.
\end{abstract}

Keywords: Yangtze River Economic Belt; carbon emissions; cultivated land use efficiency; Tobit model

Received: 2019-04-25 Accepted: 2019-12-29

Foundation: National Natural Science Foundation of China, No.71947071, No.71904151, No.71774066; Hong Kong Research Grant Council, No.ECS27604016; Financially Supported by Self-Determined Research Funds of CCNU from the Colleges' Basic Research and Operation of MOE, No.CCNU19TD004.

Author: Luo Xiang (1978-), Associate Professor, specialized in regional economics and development of economics. E-mail: philiplaw@163.com

"Corresponding author: Wan Qing (1989-), PhD, specialized in economic geography and spatial economics. E-mail: wanqing1989@126.com 


\section{Introduction}

As one of the most basic means of agriculture, cultivated land (CL) provides the basis for human survival, reproduction, and development. However, the rapid growth of the world's population has put tremendous pressure on the limited CL resources (Yang et al., 2000; Deng et al., 2005; Jin et al., 2017). In recent years, the CL resources in China have faced the problems of low quantity, low quality, low utilization efficiency, and insufficient reserve resources (Deng et al., 2015; Jin et al., 2013). In the 20th century, Brown (1995) asked who will feed China, believing that China's food shortage would lead to an overall increase in world food prices, which would bring about a global crisis. However, in the 21 st century China has paid much attention to planting high-yield varieties and constantly improving its agricultural infrastructure and technology. With less than $10 \%$ of the world's CL, China has succeeded in feeding more than $20 \%$ of the world's total population, effectively protecting China's food security (Lyle et al., 2015; Huang et al., 2004; Chen et al., 2011). In addition, China has formulated relevant policies for the strict control of the land minimum concerning $\mathrm{CL}$ and intensive utilization of land resources, and measures for the remediation of farmland and land reclamation have been proposed (Liu et al., 2017; Jin et al., 2019).

However, with the continuous improvement in fertilization and irrigation technology for farmland, CL in China is facing new risks and challenges (Feng et al., 2014; Skevas et al., 2014). The use of pesticides, fertilizers, agricultural plastic sheets, and other products may lead to various environmental spillover effects, making the utilization of farmland in China more unstable and riskier. Increasing risks in CL production will seriously threaten national food security and stable operation of the national socio-economic system (Buyanovsky et al., 1998). The Intergovernmental Panel on Climate Change Fifth Assessment Report (IPCC AR5) pointed out that rising greenhouse gas concentrations will cause frequent temperature changes, which will seriously affect the growth of food crops. Many researchers believe that the rapid development of production using agricultural land as a carrier is an important cause of accelerated global warming (Liu et al., 2015). Therefore, to effectively enhance the output-input ratio in CL use (CLU), top priority should be given to the sustainable development of agriculture, and the evaluation of agricultural land use efficiency should not only focus on economic input and output but also take into account the social and ecological functions of the agro-ecosystem (Dalgaard et al., 2001).

CLU efficiency (CLUE) is an important indicator for measuring the input-output ratio in CLU. In recent years, research in China and abroad has explored CLUE fully, and much related work has been conducted on its evaluation methods and influencing factors and on the spatiotemporal differentiation of agricultural land use efficiency at different levels (Ye et al., 2011; Quaye et al., 2010; Wang et al., 2015). At the national level, Zhang (2015) examined China's provinces (except Hong Kong, Macao, and Taiwan), constructed an indicator system from two dimensions of input and output, and used an improved data envelopment analysis (DEA) model to measure the CLUE from 1994 to 2012.The exploratory spatial data analysis (ESDA) correlation model and the rescaled range analysis $(\mathrm{R} / \mathrm{S})$ fractal model were used to analyze the differentiation characteristics of the spatiotemporal pattern of interprovincial CLUE; this revealed the driving mechanism of the spatiotemporal differentiation via the Tobit regression model. At the provincial level, Wang (2013) calculated the CLUE in Henan province based on data from 1999 to 2008 and found that the CL area remained essentially 
stable in this decade, and population density, urbanization rate, per capita gross domestic product (GDP), multi-cropping index, and fertilizer rate per hectare were the dominant factors influencing utilization efficiency of the CL resource. At present, the CLUE in China is assessed mainly at national or provincial level. The decision-making unit is a province or a city, and relatively few studies have been based on the units of urban agglomerations and economic belts (Song et al., 2014). In addition, when constructing the input-output model of CLUE, most studies have considered only economic inputs and outputs and ignored the importance of environment and ecosystem ( $\mathrm{Li}$ et al., 2013). However, related research has shown that the latter is even more important than economic output (Zhang, 2008; Chabi-Olaye et al., 2005).

To date, research on CLUE in China and abroad has achieved remarkable results in different aspects, such as methods and perspectives. Yang (2011) used the DEA-Tobit two-step method to calculate the CLUE in Shaanxi province using the Banker-Charnes-Cooper (BCC) model and analyzed the factors influencing the results using the Tobit model.He found that the electrical power of agricultural machinery, the effective irrigation rate, the crop area, the per capita GDP, and policy dummy variables had significant effects on the CLUE in the province. Ye et al. (2011) studied the CLUE for each province per year and its spatio-temporal variations using the stochastic frontier production function method, which is based mainly on the basic principles of the Battese and Coelli models (1992) and uses the log-type Cobb-Douglas production function to determine the CLUE. Currently, the most common way to measure the CLUE is by applying the DEA model. In the DEA methodology, the most widely used model should be the CCR model named after Charnes, Cooper, and Rhode (1978) and the BCC model named after Banker, Charnes, and Cooper (1984). However, the traditional DEA methodology adopts the measurement of the radial angle without considering the problem of slack variables. On this basis, Tone (2001) proposed a slack-based model (SBM) of the DEA, which not only improves the accuracy of measurement and compatibility with other measurement methods but also effectively promotes research on the utilization of CL. Based on previous research, many researchers have included undesirable outputs in consideration of both energy and environment and used the SBM to calculate the CLUE more accurately. Also, the ways and means of improving the input-output structure of CLU have been elaborated (Li et al., 2014; Zhang et al., 2017).

In recent years, with the improvement in the levels of consumption and the rapid expansion of exports, carbon emissions (CEs) in China have been increasing (Pan et al., 2008; Guo et al., 2012; Liu et al., 2015). In 2007, China surpassed the United States to become the country with the highest $\mathrm{CO}_{2}$ emissions (Gregg et al., 2008). Yao et al. (2015) pointed out that China's primary energy consumption reached 2852.36 Mtoe in 2013, accounting for $22.41 \%$ of the world's total, and China consumed $50.31 \%$ of the world's total coal, exhibiting a typical coal-dominant energy consumption pattern. Based on this situation, the Chinese government is committed to reducing CEs in 2020 by $40 \%-45 \%$ as compared with those in 2005. The increase in $\mathrm{CO}_{2}$ is one of the main causes of the greenhouse effect, and human activities are the main pathway for $\mathrm{CO}_{2}$ production (Paustian et al., 1998). Agriculture is the main source of greenhouse gases. In the process of arable land utilization, not only do the actions of plowing, irrigation, and fertilization directly produce $\mathrm{CO}_{2}$ emissions, but also indirect agricultural practices such as the production of agricultural plastic sheets and the transformation of agricultural land use generate a large amount of $\mathrm{CO}_{2}$ gas (Lee et al., 2006; 
Johnson et al., 2007).

The Yangtze River Economic Belt (YREB) is an important grain production area in China, where the annual total amount of grain produced can reach more than $30 \%$ of the country's total. This area is one of China's three major food production bases, the other two being the Northeast China Plain and the North China Plain (Xu et al., 2019). Although its strategic position has been enhanced continuously, the ecological environment and CL protection of the YREB have faced serious crises in recent years, and the problem of excessive greenhouse gas emissions is one of them (Chen et al., 2017; Li et al., 2019). In the present paper, the CEs from CL are included as an undesirable output when examining the CLUE. This paper calculated the CLUE for 11 provinces and cities in the YREB from 2007 to 2016 by SBM with CEs as undesirable output, and then the kernel density was mapped to analyze the spatiotemporal variations of CLUE. Finally, the Tobit model was used to analyze the factors affecting the CLUE. Inclusion of CEs as an undesirable output to calculate the CLUE is a new way to further improve the land use efficiency and consolidate the strength of the YREB.

\section{Study area and methods}

\subsection{General profile of the study area}

The Yangtze River Economic Belt (YREB) refers to the provinces and cities distributed along the Yangtze River. According to the Guiding Opinions of the State Council on Promoting the Development of the Yangtze River Economic Belt Based on the Golden Waterway issued by the State Council on September 25, 2014, the YREB covers 11 provinces and cities, including Shanghai, Jiangsu, Zhejiang, Anhui, Jiangxi, Hubei, Hunan, Chongqing, Sichuan, Yunnan, and Guizhou, and with an area of about 2.05 million square kilometers. The main branch of the Yangtze River flows from west to east and traverses the central part of China. The maximum difference in elevation can reach $6000 \mathrm{~m}$, and the elevation decreases gradually from west to east (Figure 1). Based on the geographical location, resource environment, economic development, and other factors, the Yangtze River can be divided into three regions, namely upstream, midstream, and downstream. The upper reaches of the Yangtze River include Guizhou, Yunnan, Sichuan, and Chongqing, its middle reaches comprise Hunan, Hubei, and Jiangxi, and its downstream part includes four provinces and the cities of Anhui, Zhejiang, Jiangsu, and Shanghai. In September 2016, the Outline of the Yangtze River Economic Belt Development Plan was officially issued, and the YREB was established as "one axis, two wings, three poles and multiple spots," which clearly defines the new direction for the construction of the YREB. It can also be anticipated that China will build a region comprising a distribution center with great comprehensive strength and that relies on the Yangtze River Golden Waterway. As one of the most active and developed areas in China, the elements such as technology and talent can flow freely within the YREB, and developing the YREB has great potential. The development of a basin economy is a strategic choice for some countries that are in the process of modernization (Zhang et al., 2010; Jin et al., 2018). In addition, the YREB is one of the main grain production regions of China, with a CL area accounting for more than $30 \%$ of the total in the country. Large lakes and rivers such as the Yangtze River, Taihu Lake, Dongting Lake, and Poyang Lake are located in the 
YREB, providing rich water resources and an ecological environment for agricultural and economic development. Therefore, it is of great practical significance to study the spatiotemporal variations of the CLUE in the YREB.

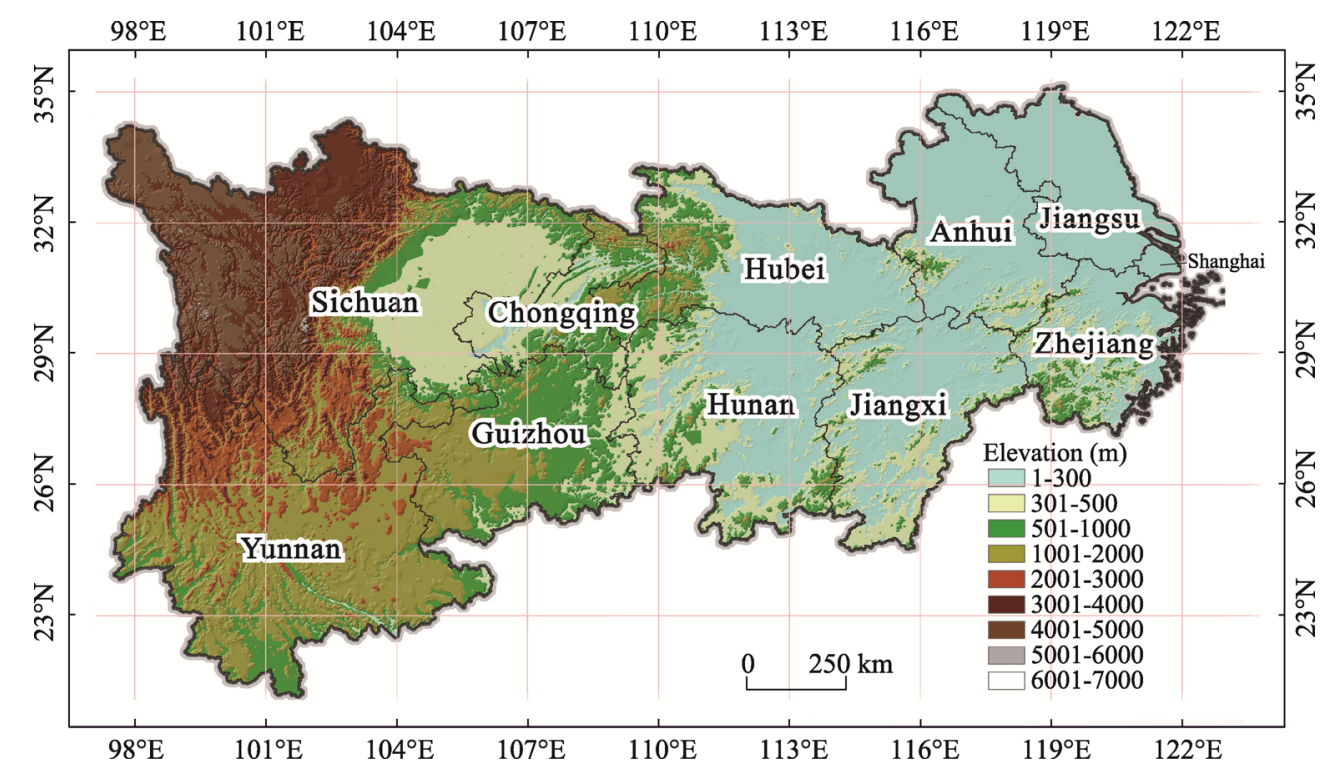

Figure 1 Geographical location of the Yangtze River Economic Belt (YREB)

The Yangtze River is the longest river in China, and the YREB spans its three major regions of eastern, central, and western parts, which offer unique advantages and tremendous development potential. In 2016, the population of the YREB reached 629 million, accounting for $45.47 \%$ of the country's total. The regional GDP reached 33718.194 billion yuan, or $45.5 \%$ of the country's total, making it the one with the greatest comprehensive strength in China. In addition, the YREB is also one of the most important food production regions in China; the total grain output (231 million tons in 2016) accounts for $37.5 \%$ of the national total. However, the YREB is currently facing the dilemma of having uncoordinated economic development and ecological protection plans. The total discharge of wastewater in the YREB in 2016 reached 31.4 billion tons, accounting for $44 \%$ of the national total. In this regard, the State Council issued the "Environmental Protection Plan for the Yangtze River Economic Belt" in 2017, and pointed out that while promoting the development of the YREB, it was mandatory to set ecological protection as the priority and stick to a green and low-carbon sustainable development model. The YREB is one of the important areas receiving strategic support in China. Against this background, the present study analyzes the spatiotemporal variations of CLUE by including CEs as an output factor and explores the variation characteristics and factors influencing CLUE in the YREB from a low-carbon perspective. Our findings may help to ensure national food security as well as provide some clues for how to implement an environmentally friendly economic development model.

\subsection{Data and indicators}

2.2.1 Indicator system for measuring the efficiency of cultivated land use

The CLUE is a measure that is used to evaluate the comprehensive utilization level of various input resources as determined by input and output indicators. Lin et al. (2017) believed 
that when constructing the input indicator system, the input factors for agricultural production should include CL endowment, labor, agricultural machinery, fertilizer, and pesticides. Therefore, the input resources for all aspects of CLU should be taken into account when setting up the indicator system. According to previous studies, the input indicator system for CL is mainly considered from three aspects: land input, labor input, and material input (Li et al., 2013; Wang et al., 2013, 2015). Specifically, the following seven input indicators were selected: (i) crop planting, $\mathrm{I}_{1}\left(10^{3} \mathrm{hm}^{2}\right)$; (ii) the number of laborers in primary industry, $\mathrm{I}_{2}$ $\left(10^{4}\right)$; (iii) the total electrical power consumption of agricultural machinery, $\mathrm{I}_{3}\left(10^{4} \mathrm{~kW}\right)$; (iv) the amount of agricultural fertilizers applied, $\mathrm{I}_{4}\left(10^{4} \mathrm{t}\right) ;(\mathrm{v})$ the use of pesticides, $\mathrm{I}_{5}(\mathrm{t})$; (vi) the amount of agricultural plastic film used, $\mathrm{I}_{6}(\mathrm{t})$; and (vii) the effective irrigation area, $\mathrm{I}_{7}$ $\left(10^{3} \mathrm{hm}^{2}\right)$. The two main output indicators were the desirable output and the undesirable output. The desirable output mainly concerns the economic and agricultural aspects with the desirable output indicators including the total output values for agriculture, forestry, animal husbandry, and fisheries, $\mathrm{O}_{1}\left(10^{8}\right.$ yuan) and total grain output, $\mathrm{O}_{2}\left(10^{4} \mathrm{t}\right)$. The undesirable output indicators mainly comprise total $\mathrm{CEs}, \mathrm{O}_{3}\left(10^{4} \mathrm{t}\right)$, including the following six categories: (i) loss of organic carbon caused by agricultural tillage; (ii) CEs from agricultural machinery operations; (iii) CEs from manufacturing and use of agricultural fertilizers; (iv) CEs from manufacturing and use of pesticides; (v) CEs from manufacturing and use of agricultural plastic sheets; and (vi) indirect generation of CEs via activities such as farmland irrigation and electricity consumption. The total amount of CEs is calculated by $E=\sum E_{i}=\sum T_{i} \cdot \delta_{i}$, where $T_{i}$ and $\delta_{i}$ are the values of each carbon source and the CE coefficient, respectively. The specific relevant CE indicator coefficients are given in Table 1.

Table 1 Carbon emission (CE) coefficients of major carbon sources arising from cultivated land use (CLU)

\begin{tabular}{lccl}
\hline \multicolumn{1}{c}{ Source } & Coefficient & Unit & Reference \\
\hline Tillage & 312.6 & $\mathrm{~kg} / \mathrm{km}^{2}$ & Wu et al., 2007 \\
Machinery & 0.18 & $\mathrm{~kg} / \mathrm{kW}$ & West et al., 2002 \\
Fertilizers & 0.8956 & $\mathrm{~kg} / \mathrm{kg}$ & West et al., 2002 \\
Pesticides & 4.9341 & $\mathrm{~kg} / \mathrm{kg}$ & Post et al., 2000 \\
Plastic sheets & 5.18 & $\mathrm{~kg} / \mathrm{kg}$ & Li et al., 2011 \\
Irrigation & 25 & $\mathrm{~kg} / \mathrm{hm}^{2}$ & Li et al., 2011 \\
\hline
\end{tabular}

\subsubsection{Indicators of factors influencing cultivated land use efficiency}

When selecting CLUE indicators, Li et al. (2011) measured the intensity of CLU in Shandong province based on five aspects, namely multi-cropping index, labor intensity, capital intensity, land productivity, and labor productivity. Wang et al. (2013) pointed out that the factors influencing CLUE include population density, urbanization rate, per capita GDP, multi-cropping index, and per hectare fertilizer rate. The main factor that influences grain output has been converted from a per hectare fertilizer basis to the quantity and quality of labor per hectare. Based on the above research and in full consideration of the effectiveness and availability of data, the following indicators were selected to construct the indicator system for CLU. 1) From the perspective of CL characteristics, the per capita CL area (PC) for each province and city in the YREB was selected as an indicator. 2) From the perspective of the development level of the study area, the per capita GDP (PG) and the primary industry 
product (PP) were selected as indicators. 3) From the perspective of the level of technological development, the electrical power consumption for agricultural machinery (MP) per hectare and the number of agricultural technicians per 10,000 people (AT) were selected as indicators. 4) From the perspective of policy, the per capita environmental pollution control investment (PI) was selected as an indicator.

\subsubsection{Data sources}

In the input indicator system for CLUE, most of the data were derived from the China Rural Statistical Yearbook (2008-2017). The number of laborers in primary industry was derived from the statistical yearbooks of the provinces and cities in the YREB from 2008 to 2017. In analyzing the factors influencing the CLUE, the following data sources were also consulted: the Land Survey Results Sharing Application Service Platform (http://tddc.mlr.gov.cn/to_Login), the China Science and Technology Database in the EPS data platform (http://olap.epsnet. com.cn/), and the China Environmental Protection Database (http://hbk.cei.cn/aspx/default. aspx). The specific data sources are listed in Table 2.

Table 2 Indicators and data sources

\begin{tabular}{|c|c|c|}
\hline & Indicators & Data sources \\
\hline \multirow[t]{7}{*}{ Input } & $\mathrm{I}_{1}$ & China Rural Statistical Yearbook (2008-2017) \\
\hline & $\mathrm{I}_{2}$ & China Rural Statistical Yearbook (2008-2017) \\
\hline & $\mathrm{I}_{3}$ & China Rural Statistical Yearbook (2008-2017) \\
\hline & $\mathrm{I}_{4}$ & China Rural Statistical Yearbook (2008-2017) \\
\hline & $\mathrm{I}_{5}$ & China Rural Statistical Yearbook (2008-2017) \\
\hline & $\mathrm{I}_{6}$ & China Rural Statistical Yearbook (2008-2017) \\
\hline & $\mathrm{I}_{7}$ & China Rural Statistical Yearbook (2008-2017) \\
\hline \multirow[t]{3}{*}{ Output } & $\mathrm{O}_{1}$ & China Rural Statistical Yearbook (2008-2017) \\
\hline & $\mathrm{O}_{2}$ & China Rural Statistical Yearbook (2008-2017) \\
\hline & $\mathrm{O}_{3}$ & $\begin{array}{l}E=\sum E_{i}=\sum T_{i} \cdot \delta_{i} \text {, where } T_{i} \text { and } \delta_{i} \text { are the values of each carbon source } \\
\text { and the CE coefficient, respectively. }\end{array}$ \\
\hline \multirow{6}{*}{$\begin{array}{l}\text { Influencing } \\
\text { factors }\end{array}$} & $\mathrm{PC}$ & Land Survey Results Sharing Application Service Platform \\
\hline & PG & $\begin{array}{l}\text { Statistical yearbooks of the provinces and cities in the YREB from } \\
2008 \text { to } 2017\end{array}$ \\
\hline & PP & $\begin{array}{l}\text { Statistical yearbooks of the provinces and cities in the YREB from } \\
2008 \text { to } 2017\end{array}$ \\
\hline & MP & $\begin{array}{l}\text { Statistical yearbooks of the provinces and cities in the YREB from } \\
2008 \text { to } 2017\end{array}$ \\
\hline & AT & EPS data platform \\
\hline & PI & China Environmental Protection Database \\
\hline
\end{tabular}

\subsection{Methods}

2.3.1 Cultivated land use efficiency model

First proposed by Tone (2001) on the basis of the traditional DEA model, the SBM solved the problem that the radial model does not contain slack variables for inefficiency measurements. The SBM is very closely related to the CCR and BCC models. The calculation principle of the SBM-undesirable model is to incorporate both the desirable and undesirable outputs 
into the measurement model of efficiency. A decision unit uses $N$ inputs $x=\left(x_{1, \cdots,} x_{N}\right) \in R_{N}^{+}$, which can produce $M$ kinds of expected outputs $y=\left(y_{1, \cdots,} y_{M}\right) \in R_{M}^{+}$and $I$ kinds of unexpected outputs $b=\left(b_{1, \ldots}, b_{I}\right) \in R_{I}^{+}$. Considering each province as a decision-making unit (DMU) and assuming that there are $\operatorname{KDUMs}(k=1, \cdots, K)$, the SBM-undesirable model is expressed as

$$
\min \rho \frac{1-\frac{1}{N} \sum_{n=1}^{N} \frac{s_{n}^{x}}{x_{n 0}}}{1+\frac{1}{M+I}\left(\sum_{m=1}^{M} \frac{s_{m}^{y}}{x_{m 0}}+\sum_{i=1}^{I} \frac{s_{i}^{b}}{x_{i 0}}\right)} \text { s.t. }\left\{\begin{array}{l}
\sum_{k=1}^{K} z_{k} x_{k n}+s_{n}^{x}=x_{n 0}, n=1, \cdots, N ; \\
\sum_{k=1}^{K} z_{k} y_{k m}+s_{m}^{y}=y_{m 0}, m=1, \cdots, M ; \\
\sum_{k=1}^{K} z_{k} b_{k i}+s_{i}^{b}=b_{i 0}, i=1, \cdots, I ; \\
z_{k} \geqslant 0 ; s_{n}^{x} \geq 0 ; s_{m}^{y} \geqslant 0 ; s_{i}^{b} \geqslant 0 .
\end{array}\right.
$$

where $\left(s_{n}^{x}, s_{m}^{y}, s_{i}^{b}\right)$ represents the slack values of input redundancy, inadequate desirable output, and redundant undesirable output. The addition of the constraint of $\sum_{k=1}^{K} z_{k}, \rho<1$ indicates that the DMU is inefficient (i.e., efficiency loss). To understand the source of inefficient CLU in each DMU, the inefficient value of CLU is decomposed as

$$
I E_{x}=\frac{1}{N} \sum_{n=1}^{N} \frac{s_{n}^{x}}{x_{n 0}} ; I E_{y}=\frac{1}{M+I} \sum_{m=1}^{M} \frac{s_{m}^{y}}{y_{m 0}} ; I E_{b}=\frac{1}{M+I} \sum_{i=1}^{I} \frac{s_{i}^{b}}{x_{i 0}}
$$

where $I E_{x}$ is the inefficiency due to input redundancy, $I E_{y}$ is that due to inadequate desirable output, and $I E_{b}$ is that due to redundant undesirable output.

Equation 3 can be used to calculate the reducible ratio of the input and undesirable output variables of the DMU from the perspective of input and output, and the expandable ratio of the desirable output variable, namely

$$
P_{1}=\frac{s_{n}^{x}}{x_{n 0}} ; P_{2}=\frac{s_{m}^{y}}{y_{m 0}} ; P_{3}=\frac{s_{i}^{b}}{x_{i 0}}
$$

where $P_{1}$ is the reducible ratio of the $n$-th input of the DMU, $P_{2}$ is the expandable ratio of the desirable output of the $m$-th item of the DMU, and $P_{3}$ is the reducible ratio of the undesirable output of the $i$-th item of the DMU.

\subsubsection{The Tobit model}

The Tobit model is a type of model in which the dependent variable has roughly continuous positive values but contains a part of the observation values with a positive probability of zero. Tobin (1958) performed probit regression on the consumption of durable goods, and probit regression is now widely used in various types of research. Given that the model parameters with censored data are estimated by the least-squares method and the estimators are inconsistent, the Tobit model was used in the present study for regression analysis when examining the factors influencing CLUE. The basic structure of the Tobit model is

$$
y_{i t}=\left\{\begin{array}{l}
\beta^{T} x_{i t}+\varepsilon_{i t}\left(\beta^{T} x_{i t}+\varepsilon_{i t}>0\right) \\
0
\end{array}\right.
$$


where $y_{i t}$ is the observed dependent variable, $x_{i t}$ is the independent variable, $\beta^{T}$ is the parameter vector to be estimated, and $\varepsilon_{i t} \sim N\left(0, \delta^{2}\right)$.

After selecting six indicators as the factors influencing CLUE-namely (i) per capita CL area (PC), (ii) per capita GDP (PG), (iii) primary industrial product (PP), (iv) electrical power consumption of agricultural machinery (MP) per hectare, (v) number of agricultural technicians per 10,000 people (AT), and (vi) per capita environmental pollution control investment $(\mathrm{PI}$ - - the following regression model was constructed according to the basic principles of the Tobit model:

$$
C E_{i t}=c+\beta_{1} P C_{i t}+\beta_{2} P G_{i t}+\beta_{3} P P_{i t}+\beta_{4} M P_{i t}+\beta_{5} A T_{i t}+\beta_{6} P I_{i t}+\varepsilon_{i t}
$$

where $C E$ is the CLUE of the provinces and cities in the YREB in the SBM, $c$ is a constant term, $\varepsilon_{i t}$ is a random error term, $t$ is the year from 2007 to 2016, and $i$ represents different provinces and cities within the YREB.

\subsubsection{Normalization}

Because of the influences of socio-economic development, geographical location, administrative area, and other factors, CEs differ greatly among provinces and cities. To better evaluate the spatiotemporal variations of CEs in the YREB as a whole, the data must be normalized before being analyzed, that is, the exponential processing of the statistical data. There are many methods for data normalization, with min-max normalization and Z-score normalization being commonly used. Min-max normalization is a linear transformation of the original data and was adopted in the present study. The formula is

$$
x^{\prime}=\frac{x-x_{\min }}{x_{\max }-x_{\min }}
$$

where $x$ is a raw datum, $x_{\max }$ is the maximum value in that year, $x_{\min }$ is the minimum value in that year, and $x^{\prime}$ is the normalized value.

\section{Results and discussion}

\subsection{Analysis of spatiotemporal variations of carbon emissions}

According to the $\mathrm{CE}$ sources and the $\mathrm{CE}$ coefficients presented in Table 1, combined with the CE calculation formula $E=\sum E_{i}=\sum T_{i} \cdot \delta_{i}$, the utilization coefficients for CL in the 11 provinces and cities in 2007-2016 in the YREB were calculated. The specific CEs from CLU are given in Table 3, from which the following can be seen. 1) From the perspective of the YREB as a whole, the total CEs from CLU showed an upward trend before 2014 and decreased gradually thereafter. At province and city level, the CEs for CL also showed a rise at first and then a slowly decreasing trend. The turning points occurred around 2013-2014, suggesting that in the YREB, the CEs from CL in various provinces and cities showed the same trend over time. 2) In 2007, the CEs from CL in Jiangsu province were the highest (4,082,200 tons). By 2016, the province with the highest CEs from CL in the whole year was Anhui province (4,013,600 tons). However, between 2007 and 2016, the region with the highest average CEs was Hubei province, with 4,119,500 tons. The above three provinces are concentrated in the southeastern part of the YREB and show a certain spatial agglomera- 
tion. The region with the lowest average CEs was Shanghai, with only 424,400 tons. The main reason for this is that Shanghai has the smallest urban area as well as the smallest CL area in the YREB compared with the other regions; also the agricultural industrial production of Shanghai accounts for only a small proportion of its gross regional product. 3) The CEs for provinces and cities in the YREB tended to be unified over time, with average CEs over three million tons accounting for nearly half of the total number of provinces and cities. These were Jiangsu, Anhui, Hubei, Hunan, and Sichuan provinces, with CEs of 4,064,400, $4,051,900,4,119,500,3,234,400$, and 3,242,300 tons, respectively.

Table 3 CEs from CLU in the Yangtze River Economic Belt

\begin{tabular}{cccccccccccc}
\hline \multirow{2}{*}{ Regions } & \multicolumn{10}{c}{ Year } & \multicolumn{1}{c}{ Average } \\
\cline { 2 - 10 } & 2007 & 2008 & 2009 & 2010 & 2011 & 2012 & 2013 & 2014 & 2015 & 2016 & \\
\hline Shanghai & 28.29 & 28.55 & 26.09 & 25.63 & 25.11 & 23.36 & 22.82 & 22.02 & 20.98 & 19.60 & 24.24 \\
Jiangsu & 408.22 & 408.29 & 415.04 & 414.50 & 412.49 & 409.01 & 405.91 & 404.10 & 396.96 & 389.90 & 406.44 \\
Zhejiang & 145.38 & 147.53 & 149.13 & 148.23 & 149.11 & 150.71 & 151.63 & 147.96 & 145.89 & 139.67 & 147.52 \\
Anhui & 374.26 & 379.77 & 386.80 & 398.38 & 410.56 & 416.34 & 425.00 & 426.57 & 423.85 & 410.36 & 405.19 \\
Jiangxi & 189.09 & 195.18 & 199.31 & 206.38 & 207.07 & 209.46 & 209.83 & 209.38 & 210.11 & 207.22 & 204.30 \\
Hubei & 373.63 & 401.05 & 413.84 & 425.33 & 429.67 & 429.97 & 422.50 & 420.44 & 406.07 & 397.02 & 411.95 \\
Hunan & 295.49 & 301.53 & 311.39 & 318.65 & 325.22 & 335.36 & 337.70 & 337.73 & 336.21 & 334.77 & 323.40 \\
Chongqing & 103.84 & 108.08 & 113.40 & 114.42 & 119.03 & 119.84 & 120.78 & 121.96 & 122.94 & 121.38 & 116.57 \\
Sichuan & 304.20 & 310.62 & 319.02 & 321.84 & 328.94 & 332.38 & 330.97 & 331.29 & 331.91 & 331.06 & 324.23 \\
Yunnan & 201.27 & 216.21 & 222.93 & 238.92 & 257.23 & 274.89 & 285.33 & 296.04 & 302.46 & 307.35 & 260.26 \\
Huizhou & 99.88 & 109.56 & 112.01 & 107.26 & 117.48 & 122.94 & 122.89 & 127.33 & 130.09 & 130.90 & 118.03 \\
Total & 2523.54 & 2606.35 & 2668.97 & 2719.56 & 2781.90 & 2824.26 & 2835.34 & 2844.84 & 2827.47 & 2789.22 & 2742.14 \\
\hline
\end{tabular}

To represent more directly the spatiotemporal evolutions of CEs from CLU for the provinces and cities in the YREB, CEs for 2007, 2010, 2013, and 2016 were selected as representative data; the data were normalized by Equation 6, and the processed results were divided into four levels, namely micro-emissions (0-0.25), mild emissions $(0.25-0.5)$, intermediate emissions (0.5-0.75), and heavy emissions (0.75-1). The ArcGIS software (version 10.2) was used to characterize spatially the differences in CEs for CL from the various provinces and cities in the YREB. The deeper colors represent higher relative CEs (Figure 2).

Figure 2 shows that with time, the overall color of the YREB gradually deepens, indicating that the CEs show certain spatial clustering and positive correlation with respect to spatial distribution. The focus of the spatial distribution of CEs in the YREB shifted from the southeast to an equilibrium position involving the east and the west, and the mild emission areas in the west gradually shifted to the intermediate emission areas; while the intermediate emission areas gradually shifted to the heavy emission areas. Overall, the regional CEs from $\mathrm{CL}$ tended to be balanced. These results showed a gradually increasing spatial agglomeration of the CE intensities for the various provinces and cities in the YREB, and that the arable land use efficiency and agricultural technology levels between adjacent cities had a certain spillover effect, and that exchange and cooperation between adjacent regions were frequent. On the whole, there was a convergence of the time dimension in the regions with high CEs in the YREB. The provinces and cities with initially high CEs had a higher probability of 
maintaining this status. By 2016, except for Shanghai, which was still characterized by micro-emissions, no provinces and cities were classified as micro-emission areas due to two reasons. First, the CEs from CL in the various provinces and cities in the YREB showed a generally increasing trend over the past 10 years. Second, secondary and tertiary industries are the pillar industries of Shanghai, and thus the CEs from CL in Shanghai were much lower than those of other areas, leading to the relatively higher CEs from CL in other areas.

(a) 2007

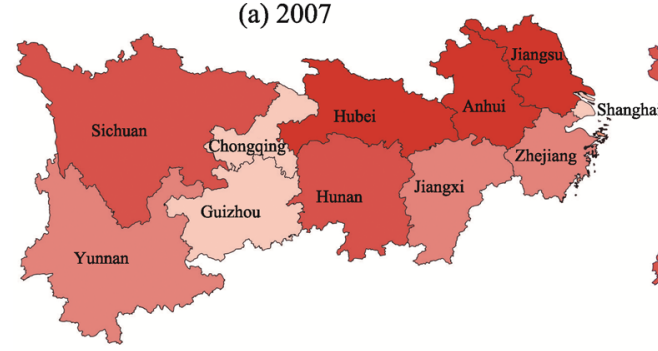

(c) 2013 (b) 2010

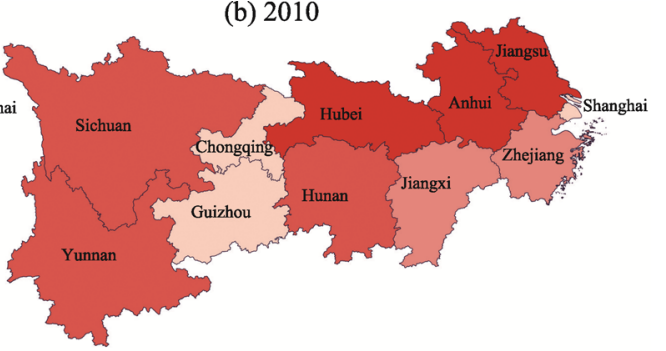

(d) 2016

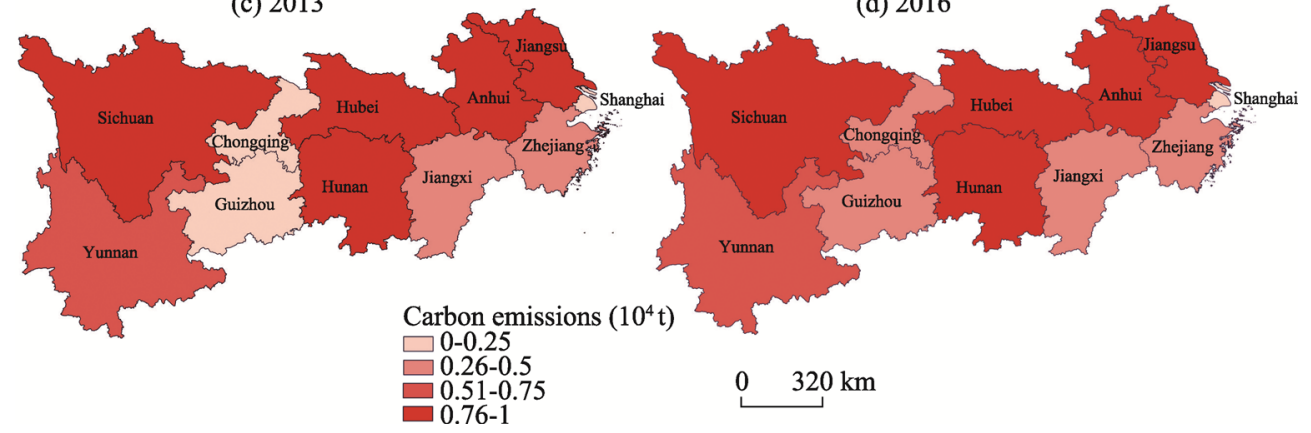

Figure 2 Spatiotemporal variations of cultivated CEs in the Yangtze River Economic Belt

\subsection{Dynamic variations of cultivated land use efficiency}

To determine how CEs affect CLUE, the CCR model and SBM with the DEA Solver Pro software (version 5.0) were used to measure the utilization rate of CL in the various provinces and cities in the YREB. In the SBM, CEs were used as an undesirable output to measure the CLUE. The results for CLUE by the CCR model and SBM for 2007, 2010, 2013, and 2016 were selected as shown in Table 4, from which the following can be seen. 1) The CLUE from the perspective of CEs can more accurately represent the comprehensive level of CLU in the local area. The CLUE is a significant indicator that reflects the level of regional agricultural economic development. The CLUE under CE constraints not only has high dependence on agricultural technology, but also fully reflects the sustainable development of CLU. After the inclusion of CEs as an undesirable output, the CLUE in the various provinces and cities in the YREB was generally reduced. 2) Regardless of whether the CCR model or SBM was used, the CLUE for the provinces and cities in the YREB showed an overall upward trend with time. Given that the DEA model is based on the relative utilization efficiency, for comparison purposes (vertical basis), it can be seen that the CLUE for some regions in 2016 attained a value of one. Compared with other provinces and cities, some regions have maintained high CLUE since 2007, and their CLUE shows a value of one in 2007 and 2013. 
Table 4 CLUE for each province or city of the Yangtze River Economic Belt in specific years

\begin{tabular}{|c|c|c|c|c|c|c|c|c|}
\hline \multirow{2}{*}{ Region } & \multicolumn{2}{|c|}{2007} & \multicolumn{2}{|c|}{2010} & \multicolumn{2}{|c|}{2013} & \multicolumn{2}{|c|}{2016} \\
\hline & CCR & SBM & CCR & SBM & CCR & SBM & CCR & SBM \\
\hline Shanghai & 0.9135 & 0.6728 & 0.9844 & 0.8853 & 1 & 1 & 1 & 1 \\
\hline Jiangsu & 1 & 1 & 0.9822 & 0.7922 & 0.9986 & 0.9338 & 1 & 1 \\
\hline Zhejiang & 0.6957 & 0.4185 & 0.7997 & 0.5749 & 0.9321 & 0.7798 & 1 & 1 \\
\hline Anhui & 0.9006 & 0.5860 & 0.9113 & 0.6419 & 0.8974 & 0.6633 & 0.9527 & 0.7483 \\
\hline Jiangxi & 1 & 1 & 0.9653 & 0.7987 & 1 & 1 & 1 & 1 \\
\hline Hubei & 0.8922 & 0.5689 & 0.9017 & 0.5717 & 0.9576 & 0.7586 & 1 & 1 \\
\hline Hunan & 0.9487 & 0.7318 & 0.9482 & 0.7720 & 0.9401 & 0.7738 & 1 & 1 \\
\hline Chongqing & 0.9905 & 0.9267 & 0.9824 & 0.9087 & 0.9974 & 0.9319 & 1 & 1 \\
\hline Sichuan & 1 & 1 & 0.9701 & 0.8881 & 0.9784 & 0.9371 & 1 & 1 \\
\hline Yunnan & 0.7185 & 0.4993 & 0.6519 & 0.4753 & 0.7441 & 0.5431 & 0.7821 & 0.5461 \\
\hline Huizhou & 1 & 1 & 0.9819 & 0.8595 & 0.8672 & 0.7141 & 1 & 1 \\
\hline
\end{tabular}

On the basis that including CEs as an undesirable output represents the CLUE more objectively, the CLUE was mainly analyzed from a low-carbon perspective. To analyze the spatiotemporal variations of CLUE in the various provinces and cities of the YREB, the years 2007, 2010, 2013, and 2016 were selected as representative time points, and a kernel density analysis was performed using the ArcGIS (version 10.2) spatial analysis software. As shown in Figure 3, different colors represent different levels of CLUE.

(a) 2007

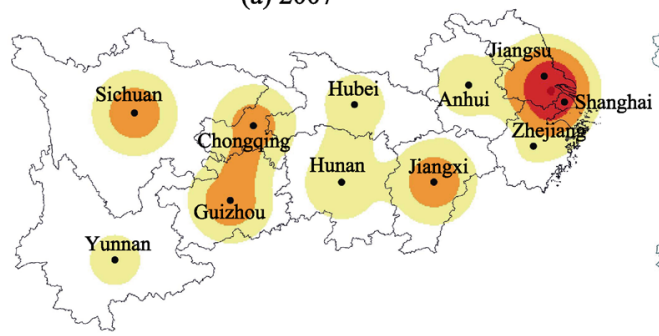

(c) 2013

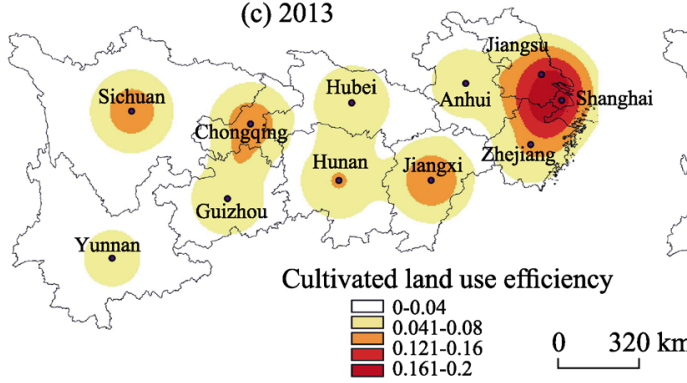

(b) 2010

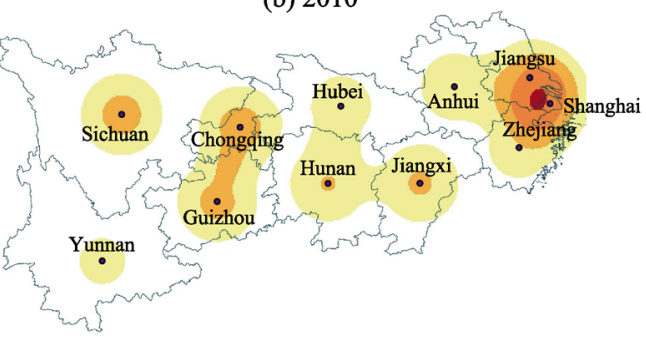

(d) 2016

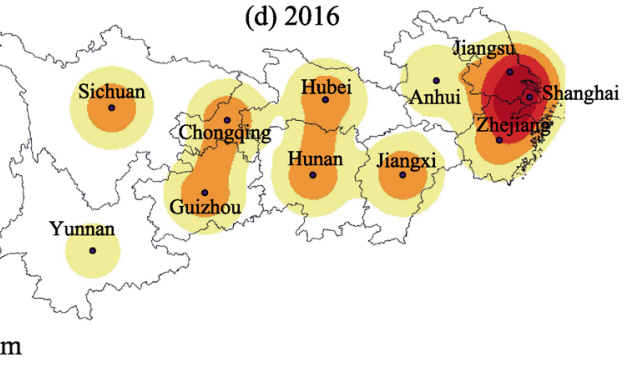

Figure 3 Kernel density map of CLU efficiency (CLUE) in the Yangtze River Economic Belt

The results in the above figures and tables can be summarized as follows.1) In the YREB, the CLUE under the constraint of CEs has spatial spillover effects and regional synergies. Specifically, the areas with high utilization efficiency for CL radiated to those with low utilization efficiency. The kernel density circle centered on Jiangsu-Shanghai in the southeast expanded year by year and gradually spread to the central area of Zhejiang province. Among all the provinces and cities, Zhejiang showed the most obvious increase (from 0.418 to 1.000) in CLUE over 10 years. 2) The CLUE for various provinces and cities in the 
YREB showed an upward trend in the time dimension, while the kernel density showed that the spatial dimension was high in the east and low in the west. High-nuclear-density areas are concentrated in the Yangtze River Delta. However, the CLUE in the middle reaches of the Yangtze River was maintained overall at equilibrium. Among the areas, Shanghai, Jiangsu, Jiangxi, Hunan, Chongqing and Sichuan were the provinces and cities with the highest CLUE. 3) In the YREB, the kernel density circle for the CLUE for Yunnan indicated that this province was always sluggish compared with the other regions. For instance, Yunnan has been displaying a lower growth rate of CLUE for a considerable time, that is, from 2007 to 2016 the CLUE only increased from 0.4993 to 0.5461 , which may be attributed to the low soil quality and the sloping nature of the CL in this region. Most of the topography of Yunnan province is mountainous or plateaus with steep slopes. Besides, the positive effects of technological progress have lagged to some extent with Yunnan's agricultural production technology being relatively underdeveloped, thus the feedback effect on the CLUE may not yet have been realized. Therefore, the local government should do more to promote CLUE in Yunnan province through policy encouragement, active prevention and control of soil erosion, and construction of terraces. 4) The CLUE in Jiangxi and Jiangsu was at relatively high levels during the study period, but there was a slight decline in the two provinces from 2007 to 2010. In 2010, the CLUE of Jiangsu province fell from 1 to 0.7922 , and that of Jiangxi province decreased from 1 to 0.7987 . According to the indicator data, the reason for this phenomenon in Jiangsu was that the CEs increased rapidly in 2007-2010 and declined slowly thereafter, which may be due to the fact that during this period, the total grain output of the province declined to some extent, but has been increasing rapidly since 2010. 5) The CLUE in Guizhou was variable, first decreasing and then increasing, thus continuous observation and monitoring are needed in this province. The government should actively encourage the uptake of scientific and technological innovations and other initiatives, and promote a steady increase in CLUE in province with the assistance of surrounding areas (i.e., Chongqing and Hubei) where there is a higher CLUE.

\subsection{Analysis of factors influencing cultivated land use efficiency}

Using the Tobit model established in Equation 5, the Stata software (version 12) was used to analyze the correlation between CLUE and the factors influencing it. The indicators include the per capita CL area (PC), the per capita GDP (PG), the primary industrial product (PP), the electrical power consumption of agricultural machinery (MP) per hectare, the number of agricultural technicians per 10,000 people (AT) and the per capita environmental pollution control investment (PI). The results of the regression analysis are given in Table 5.

Table 5 Regression results of CLUE using the Tobit model

\begin{tabular}{ccccc}
\hline Variables & Coefficient & Std. Err. & $Z$ & Significance \\
\hline PC & -0.0004409 & 0.0001838 & -2.4 & 0.016 \\
PG & $3.14 \mathrm{E}-06$ & $1.08 \mathrm{E}-06$ & 2.9 & 0.004 \\
PP & 0.0000704 & 0.0000279 & 2.52 & 0.012 \\
MP & -0.0208301 & 0.0070656 & -2.95 & 0.003 \\
AT & 0.0573215 & 0.0258476 & 2.22 & 0.027 \\
PI & $-1.16 \mathrm{E}-06$ & 0.0000392 & -0.03 & 0.976 \\
\hline
\end{tabular}


From the regression results, the PC, PG, PP, MP, and AT all passed the 5\% statistical significance test, among which PC and MP passed the 1\% statistical significance test. However, PI did not pass any statistical significance test, indicating that it had no significant effect on the CLUE.

The CL endowment, the economic development level and agricultural technology input for an area can significantly affect the local CLUE. 1) From the perspective of CL endowment, the per capita CL area had a significant negative impact on CLUE, indicating that controlling the CL area to a certain extent can effectively enhance the CLUE. This result indirectly reflects the possible fact that the intensity of CLU in the YREB was still not high enough, and that, despite there being extensive cultivation of large areas of land, the production capacity of CL may be rather low. The government should make more efforts to promote farmland consolidation and intensify the cultivation of land, which may help to effectively improve the CLUE. 2) The level of economic development in a region also had a significant impact on the CLUE. The significance level between per capita GDP and CLUE in the provinces and cities of the YREB was 0.004 , and that between the primary industrial output value and the CLUE was 0.012 , both of which pass the significance level test of 0.05 and are positively correlated. The per capita GDP is one of the important indicators to measure the level of economic development, and the primary industrial product can reflect the development level of local primary industry. Generally, economic development improves mainly the CLUE by promoting modern technology, but technological progress can also play a negative role with respect to the intensity of CEs. However, for underdeveloped areas, it will take a long time for technological progress to show reducing effects on CEs. 3) From the perspective of the relevant technical level, the number of agricultural technicians can have a significant positive impact on the CLUE, while the electrical power consumption of agricultural machinery per hectare has a negative impact. In other words, an increase in the number of agricultural technicians can realize an improvement of local CLUE. For example, every $1 \%$ increase in the number of agricultural technicians will increase the CLUE by $0.057 \%$. The electrical power consumption of agricultural machinery per hectare is negatively correlated with the CLUE, indicating that the current mechanized production mode does not fully exploit the potential of the CL. This phenomenon may be attributable to the fragmentation and dispersion of CL in the YREB. To improve the CLUE in the region corresponding to realizing high output and low input, the government must actively encourage the recruitment of relevant technical professionals to promote improvements in agricultural technology. 4) From the perspective of policy factors, there was no significant correlation between the per capita investment in environmental pollution control and the CLUE. However, the urbanization process can have a major impact on the quantity and quality of CL. Thus, the government should actively formulate policies related to agricultural land consolidation and protection to ensure the sustainable use of CL resources and develop a green and low-carbon management system for CLU.

\section{Conclusions}

This paper has examined the YREB as a research unit and used the SBM-undesirable model to measure the CLUE of the provinces and cities in the study area by including CEs as an undesirable output. The results were compared with those obtained by the CCR model. In 
addition, kernel density mapping was used to analyze the dynamic variations of CLUE in the provinces and cities of the YREB and determine the factors influencing CLUE. The following conclusions can be drawn.

1) In the YREB, the CEs for $C L$ at first showed a rise and then exhibited a slowly decreasing trend. In 2007, the CEs for CL in the YREB were 25.2354 million tons, and gradually increased to 28.44 million tons in 2014. After 2014, the CEs for CL decreased year by year and fell to 27.8922 million tons in 2016, indicating that the government's CE reduction measures had achieved a certain level of impact. In the YREB, the CEs from CL in various provinces and cities overlapped to some extent and showed the same trend over time. Between 2007 and 2016, the region with the highest average CEs was Hubei province, with average CEs of 4,119,500 tons. The region with the lowest average CEs was Shanghai, with an average annual amount of only 424,400 tons. The main reason for this is that the city's urban area is the smallest in the YREB, and its agricultural industrial production accounts for only a small proportion of its GDP.

2) The CLUE from the perspective of CEs can more accurately represent a comprehensive assessment of the level of CLU in the local area. The CLUE is a significant indicator that reflects the level of regional agricultural economic development. The CLUE under the constraint of CEs not only has a high dependence on agricultural technology, but also considers the CLUE from an ecological perspective. Previous research has mostly focused on favorable and desirable outputs, and often ignored the undesirable outputs caused by excessive energy consumption. The evaluation of CLUE which takes into consideration CEs not only directly reflects the input-output ratio of CLU, but also takes into account the external effects and sustainable utilization from an ecological perspective.

3) The CLUE of various provinces and cities in the YREB showed an upward trend in the time dimension, while the kernel density maps showed that the spatial dimension was high in the east and low in the west. High-nuclear-density areas were concentrated in the Yangtze River Delta. In the YREB, the CLUE under carbon constraints had spatial spillover effects and regional synergies: the kernel density circle centered on Jiangsu-Shanghai in the southeast gradually radiated to the surrounding $\mathrm{CL}$ which were areas with low utilization efficiency. The CLUE in Zhejiang showed the most obvious increase, rising from 0.418 to 1.000 over the past 10 years. Yunnan's arable land use efficiency increased slowly, only rising from 0.4993 to 0.5461 over the period 2007-2016, which may reflect the level of the local natural environment and the level of technology infrastructure in the area. The local government should promote efficiency through policy measures, including the control of soil erosion and construction of terraces.

4) The provinces and cities within the YREB show a clear trend towards equilibrium in terms of CEs and CLUE. This may be due to the fact that certain factors within the YREB were freely circulating, and the exchange of science and technology was unimpeded. The Tobit model estimates show that the per capita GDP, the primary industrial production, and the number of agricultural technicians per 10,000 people have positive effects on the CLUE. The significance level of the per capita GDP was 0.004 , and that of primary industrial production was 0.012 , both variables passing the significance test at the 0.05 level. The number of agricultural technicians per 10,000 people has a positive impact on the CLUE: every $1 \%$ increase in the number of agricultural technicians would increase the CLUE by $0.057 \%$. The per capita CL area and the electrical power consumption for agricultural machinery per hec- 
tare had significant negative impacts on the local arable land use efficiency, demonstrating that the level of urban economic development, population density and technological progress affected adversely the development of arable land use efficiency.

According to the above findings, the government should make efforts to improve the CLUE from the following two aspects. First, the government should effectively control the total amount of CEs and establish a scientific management system for CEs by increasing policy support, strengthening relevant scientific research and improving the endowment of CL resources. Second, the government should maintain a balance between economic development and ecological protection, focusing on finding an equilibrium position between the 'desirable output' and the 'undesirable output' of CL. The agricultural production of the YREB should keep pace with economic developments by actively improving the utilization of CL on a large-scale, strengthening agricultural land consolidation, rationally controlling the area of CL and improving the construction of agricultural infrastructure.

\section{Limitations and future work}

This paper incorporates CEs as an undesirable output in the indicator system and uses the DEA model to calculate more accurately the CLUE for the YREB from a new perspective. However, there are still several issues that need to be addressed. First, this study has considered comprehensively the factors that may affect the CLUE from four aspects, that is, society, the economy, the environment and government policy. These aspects are all about the technical capabilities and development levels of each region, and thus the relevant approaches for improving CLUE should also consider these aspects. However, the regions in the study are not mutually independent from other regions, and the changes in CLUE in these regions are not determined solely by internal factors, but also by influences from the adjacent provinces and cities. Hence, follow-up research should focus on the dissection of the influence of neighboring provinces and cities on the variations in CLUE in the various regions and the corresponding optimization measures. Second, when constructing the indicator system for CLUE, indicators were selected that were relatively easy to define and quantify. However, other indicators which are difficult to quantify such as the overall quality of CL resources, the degree of pollution from CL, the degree of fragmentation of CL and the farmers' willingness to participate can also impact on the CLUE. With a continuous transfer of rural labor and a deterioration of the ecological environment, the improvement of CLUE is faced with significant challenges. Future research should seek to evaluate CLUE by also addressing the aforementioned aspects.

\section{References}

Banker R D, Charnes A, Cooper W W, 1984. Some models for estimating technical and scale inefficiencies in data envelopment analysis. Management Science, 30(9): 1078-1092.

Battese G E, Coelli T J, 1992. Frontier production functions, technical efficiency and panel data: With application to paddy farmers in India. Journal of Productivity Analysis, 3: 153-169.

Brown L R, 1995. Who Will Feed China? Wake-up Call for a Small Planet. London: England Earthscan Publications.

Buyanovsky G A, Wagner G H, 1998. Carbon cycling in cultivated land and its global significance. Global Change Biology, 4(2): 131-141.

Chabi-Olaye A, Nolte C, Schulthess F et al., 2005. Relationships of intercropped maize, stem borer damage to 
maize yield and land-use efficiency in the humid forest of Cameroon. Bulletin of Entomological Research, 95(5): 417-427.

Charnes A, Cooper W W, Rhodes E, 1978. Measuring the efficiency of decision-making units. European Journal of Operational Research, 2(6): 429-444.

Chen Y J, Xiao B L, Fang L N et al., 2011. The quality analysis of cultivated land in China. Scientia Agricultura Sinica, 44(17): 3557-3564. (in Chinese)

Chen Y S, Zhang S H, Huang D S et al., 2017. The development of China's Yangtze River Economic Belt: How to make it in a green way? Science Bulletin, 62(9): 648-651.

Dalgaard T, Halberg N, Porter J R, 2001. A model for fossil energy use in Danish agriculture used to compare organic and conventional farming. Agriculture Ecosystems and Environment, 87(1): 51-65.

Deng X Z, Huang J K, Rozelle S et al., 2005. Cultivated land conversion and potential agricultural productivity in China. Land Use Policy, 23(4): 372-384.

Deng X Z, Huang J K, Rozelle S et al., 2015. Impact of urbanization on cultivated land changes in China. Land Use Policy, 45: 1-7.

Feng T, Zhang F R, Li C et al., 2014. Spatial distribution of prime farmland based on cultivated land quality comprehensive evaluation at county scale. Transactions of the Chinese Society of Agricultural Engineering, 30(1): 200-210, 293. (in Chinese)

Gregg J S, Andres R J, Marland G, 2008. China: Emissions pattern of the world leader in $\mathrm{CO}_{2}$ emissions from fossil fuel consumption and cement production. Geophysical Research Letters, 35(8): 135-157.

Guo J, Zhang Z K, Meng L, 2012. China's provincial $\mathrm{CO}_{2}$ emissions embodied in international and interprovincial trade. Energy Policy, 42(C): 486-497.

Huang J K, Deng X Z, Rozelle S, 2004. Cultivated land conversion and bio-productivity in China. Proceedings of SPIE - The International Society for Optical Engineering, 5544.

Jin G, Chen K, Wang P et al., 2019. Trade-offs in land-use competition and sustainable land development in the North China Plain. Technological Forecasting and Social Change, 141: 36-46.

Jin G, Deng X Z, Chu X et al., 2017. Optimization of land-use management for ecosystem service improvement: A review. Physics and Chemistry of the Earth, 101: 70-77.

Jin G, Deng X Z, Zhao X D et al., 2018. Spatiotemporal patterns in urbanization efficiency within the Yangtze River Economic Belt between 2005 and 2014. Journal of Geographical Sciences, 28(8): 1113-1126.

Jin G, Li Z H, Deng X Z et al., 2019. An analysis of spatiotemporal patterns in Chinese agricultural productivity between 2004 and 2014. Ecological Indicators, 105: 591-600.

Jin J J, Jiang C, Li L, 2013. The economic valuation of cultivated land protection: A contingent valuation study in Wenling City, China. Landscape and Urban Planning, 119: 158-164.

Johnson M F, Franzluebbers A J, Weyers S L et al., 2007. Agricultural opportunities to mitigate greenhouse gas emissions. Environmental Pollution, 150(1): 107-124.

Kaoru T, 2001. A slacks-based measure of efficiency in data envelopment analysis. European Journal of Operational Research, 130(3): 498-509.

Lee J, Six J, King A P et al., 2006. Tillage and field scale controls on greenhouse gas emissions. Journal of Environmental Quality, 35(3): 714-725.

Li B, Zhang J B, Li H P, 2011. Research on spatial-temporal characteristics and affecting factors decomposition of agricultural carbon emission in China. China Population, Resources and Environment, 21(8): 80-86. (in Chinese)

Li H, Shi J F, 2014. Energy efficiency analysis on Chinese industrial sectors: An improved Super-SBM model with undesirable outputs. Journal of Cleaner Production, 65: 97-107.

Li S C, Bing Z L, Jin G, 2019. Spatially explicit mapping of soil conservation service in monetary units due to land use/cover change for the Three Gorges Reservoir Area, China. Remote Sensing, 11(4): 1-21.

Li Z J, Yu X K, Gong C J, 2013. Intensity change in cultivated land use in Shandong province from 1980 to 2010. Information Engineering Research Institute, 5: 140-146.

Lin H C, Hülsbergen, Kurt-Jürgen, 2017. A new method for analyzing agricultural land-use efficiency, and its application in organic and conventional farming systems in southern Germany. European Journal of Agronomy, 83: 15-27.

Liu H G, Liu W D, Fan X M et al., 2015. Carbon emissions embodied in value added chains in China. Journal of 
Cleaner Production, 103: 362-370.

Liu X W, Zhao C L, Song W, 2017. Review of the evolution of cultivated land protection policies in the period following China's reform and liberalization. Land Use Policy, 67: 660-669.

Lyle G, Bryan B A, Ostendorf B, 2015. Identifying the spatial and temporal variability of economic opportunity costs to promote the adoption of alternative land uses in grain growing agricultural areas: An Australian example. Journal of Environmental Management, 155: 123-135.

Pan J H, Phillips J, Chen Y, 2008. China's balance of emissions embodied in trade: Approaches to measurement and allocating international responsibility. Oxford Review of Economic Policy, 24(2): 354-376.

Paustian K, Cole C V, Sauerbeck D et al., 1998. $\mathrm{CO}_{2}$ mitigation by agriculture: An overview. Climatic Change, 40(1): 135-162.

Post W M, Kwon K C, 2000. Soil carbon sequestration and land-use change: Processes and potential. Global Change Biology, 6(3): 317-327.

Quaye A K, Hall C A S, Luzadis V A, 2010. Agricultural land use efficiency and food crop production in Ghana. Environment Development and Sustainability, 12(6): 967-983.

Skevas T, Stefanou S E, Oude Lansink A, 2014. Pesticide use, environmental spillovers and efficiency: A DEA risk-adjusted efficiency approach applied to Dutch arable farming. European Journal of Operational Research, 237(2): 658-664.

Song W, Pijanowski B C, 2014. The effects of China's cultivated land balance program on potential land productivity at a national scale. Applied Geography, 46: 158-170.

Tobin J, 1958. Estimation of relationships for limited dependent variables. Econometrica, 26(1): 24-36.

Tone K, 2001. A slacks-based measure of efficiency in data envelopment analysis. European Journal of Operational Research, 130(3): 498-509.

Wang G G, Liu Y S, Li Y R et al., 2015. Dynamic trends and driving forces of land use intensification of cultivated land in China. Journal of Geographical Sciences, 25(1): 45-57.

Wang K Y, 2013. The research on impact factors and characteristic of cultivated land resources use efficiency: Take Henan province, China as a case study. Information Engineering Research Institute, 5: 2-9.

West T O, Marland G, 2002. A synthesis of carbon sequestration, carbon emissions, and net carbon flux in agriculture: Comparing tillage practices in the United States. Agriculture Ecosystems and Environment, 91(1-3): 217-232.

Wu F L, Li L, Zhang H L et al., 2007. Effects of conservation tillage on net carbon flux from farmland ecosystems. Chinese Journal of Ecology, 26(12): 2035-2039. (in Chinese)

$\mathrm{Xu}$ X B, Hu H Z, Tan Y et al., 2019. Quantifying the impacts of climate variability and human interventions on crop production and food security in the Yangtze River Basin, China, 1990-2015. Science of The Total Environment, 665: 379-389.

Yang H, Li X B, 2000. Cultivated land and food supply in China. Land Use Policy, 17(2): 73-88.

Yang S, Li S P, Luo L, 2011. Study on the farmland use efficiency and its influencing factors in Shanxi province. China Land Science, 25(2): 47-54. (in Chinese)

Yao X, Zhou H C, Zhang A Z et al., 2015. Regional energy efficiency, carbon emission performance and technology gaps in China: A meta-frontier non-radial directional distance function analysis. Energy Policy, 84: $142-154$.

Ye H, Pu L J, 2011. Study on the cultivated land use efficiency between different regions of China and its convergence. Journal of Natural Resources, 26(9): 1467-1474. (in Chinese)

Zhang J R, Zeng W H, Wang J N et al., 2017. Regional low-carbon economy efficiency in China: Analysis based on the Super-SBM model with $\mathrm{CO}_{2}$ emissions. Journal of Cleaner Production, 163: 202-211.

Zhang L, Zhang F R, An P L et al., 2008. Comparative study of cultivated land use intensive degree and its change law at different economic levels. Transactions of the Chinese Society of Agricultural Engineering, 24(1): 108-112. (in Chinese)

Zhang N, Gao Z Q, Wang X M et al., 2010. Modeling the impact of urbanization on the local and regional climate in Yangtze River Delta, China. Theoretical and Applied Climatology, 102(3/4): 331-342.

Zhang R T, Jiao H F, 2015. Spatial-temporal pattern differentiation and its mechanism analysis of using efficiency for provincial cultivated land in China. Transactions of the Chinese Society of Agricultural Engineering, 31(2): 277-287. (in Chinese) 